\title{
Diagnosis and management of trigemino-cavernous fistulas: case reports and review of the literature
}

\author{
Timothy R Miller, ${ }^{1}$ Gaurav Jindal, ${ }^{1}$ Suyash Mohan, ${ }^{2}$ Manuel Fortes, ${ }^{1}$ Robert Hurst, ${ }^{2}$ \\ Bryan Pukenas, ${ }^{2}$ Dheeraj Gandhi ${ }^{1}$
}

- Additional material is

published online only. To view please visit the journal online (http://dx.doi.org/10.1136/ neurintsurg-2013-010932).

${ }^{1}$ Department of Diagnostic Radiology and Neuroradiology, University of Maryland Medical Center, Baltimore, Maryland, USA

${ }^{2}$ Department of Diagnostic Radiology and Neuroradiology, Hospital of the University of Pennsylvania, Philadelphia, Pennsylvania, USA

\section{Correspondence to}

Dr T R Miller, Department of Diagnostic Radiology,

University of Maryland Medical

Center, Room N2W78, 22

South Greene Street, Baltimore, MD 21201, USA;

Tmiller5@umm.edu

Received 9 August 2013 Revised 6 November 2013 Accepted 15 December 2013

Published Online First

6 January 2014

\section{CrossMark}

\author{
To cite: Miller TR, Jindal $G$, \\ Mohan S, et al. J \\ Neurolntervent Surg \\ 2015;7:73-78.
}

\section{ABSTRACT}

Although usually asymptomatic, a persistent trigeminal artery (PTA) can rarely be associated with a direct fistula to the cavernous sinus (ie, trigemino-cavernous fistula). We present three patients with trigemino-cavernous fistulas; two were subsequently treated using modern endovascular techniques while the third initially declined therapy. We then review the literature of reported cases of this unusual entity. The aberrant anatomy associated with a PTA presents unique challenges to the management of these lesions, and must be well delineated prior to treatment. Finally, conservative management of trigemino-cavernous fistulas, either de novo or recurrent, may be considered if they demonstrate no evidence of cortical venous reflux and patient symptoms are tolerable.

\section{INTRODUCTION}

A persistent trigeminal artery (PTA) is the most common fetal carotid-vertebrobasilar anastomosis to persist into adult life, with an incidence of approximately $0.68 \% .^{1-4}$ The vessel appears at the 3-4 mm embryonic stage and connects the developing cavernous internal carotid arteries to the precursors of the basilar artery, the paired plexiform longitudinal neural arteries. ${ }^{1}{ }^{3}{ }^{5}$ Together with more caudal fetal carotid-vertebrobasilar anastomoses, the trigeminal artery serves to supply the hindbrain in early embryonic development before normally regressing as the posterior communicating and vertebral arteries develop. ${ }^{156}$ A PTA can be identified as an aberrant vessel originating from the cavernous internal carotid artery proximal to the meningohypophyseal trunk, which courses posteriorly to connect to the basilar artery between the anterior inferior and superior cerebellar arteries. ${ }^{5}$ The vessel can take a lateral, petrosal, or medial sphenoid course in relation to the dorsum sellae, and may give rise to pontine perforators, meningohypophyseal branches, as well as branches supplying the trigeminal nerve. ${ }^{2} 36$

The Saltzman classification characterizes PTAs based on the relative contribution of the proximal basilar artery to the more distal posterior circulation. ${ }^{2} 7$ In Saltzman type 1, the basilar artery proximal to the PTA is hypoplastic and the posterior communicating arteries are either absent or small. Here the more distal basilar artery and its branches are supplied predominately by the PTA. ${ }^{4} 7$ In Saltzman type 2, the mid-basilar is well formed and the posterior communicating arteries are present, contributing to flow distally. ${ }^{4} 67$
Finally, some PTA variants do not fall into either category, including anomalous origins of a cerebellar artery from a PTA, with or without communication to the basilar artery (box 1). ${ }^{13}$

Although most often an incidental finding, PTAs have been associated with aneurysms, subarachnoid hemorrhage, trigeminal neuralgia, cranial nerve deficits, posterior circulation thromboembolism and, rarely, direct fistula with the cavernous sinus (trigemino-cavernous fistula). ${ }^{1}{ }^{4-7}$ The aberrant vessel may be predisposed to aneurysm formation and rupture due to either structural defects in its wall or hemodynamic stresses as an arterial branch point. $^{1} 245$

We report three patients who developed trigemino-cavernous fistulas, all of whom are female, and experienced the spontaneous onset of symptoms. Two were treated with modern endovascular techniques while a third initially elected to conservatively follow her lesion before subsequently undergoing palliative embolization at an outside institution.

\section{CASE REPORTS}

\section{Case No 1}

This woman acutely developed right orbital pain, double vision, and pulsatile tinnitus following an

\section{Box 1 Saltzman classification of persistent} trigeminal arteries

Saltzman type 1

- Hypoplastic mid basilar artery proximal to insertion of a persistent trigeminal artery (PTA)

- Posterior communicating arteries are either aplastic or hypoplastic

- Superior cerebellar and posterior cerebral arteries supplied predominately by the PTA

- Saltzman type 2

- Mid basilar artery well formed

- Posterior communicating arteries present

- Posterior communicating and basilar arteries contribute to distal posterior circulation

- Saltzman type 3 or variant

- A cerebellar artery arises from the PTA, often an anterior inferior cerebellar artery

- A PTA may or may not communicate with the basilar artery 
Figure 1 Anteroposterior views of the right internal carotid and left vertebral artery angiograms $(A, B)$ demonstrate a right trigemino-cavernous sinus fistula with arteriovenous shunting into the right cavernous sinus, reflux into the ipsilateral superior ophthalmic vein (black arrow (A)), cross filing of the contralateral cavernous sinus (white arrow (A)), and venous drainage into the bilateral inferior petrosal sinuses and internal jugular veins. The persistent trigeminal artery (PTA; white arrow (B)) is only clearly delineated on vertebral artery injections due to rapid arteriovenous shunting. Following transarterial coil embolization of the right cavernous sinus, there is no evidence of residual arteriovenous shunting on lateral views of the left vertebral (C) and right internal carotid (D) artery angiograms (white arrow indicating residual PTA stumps).

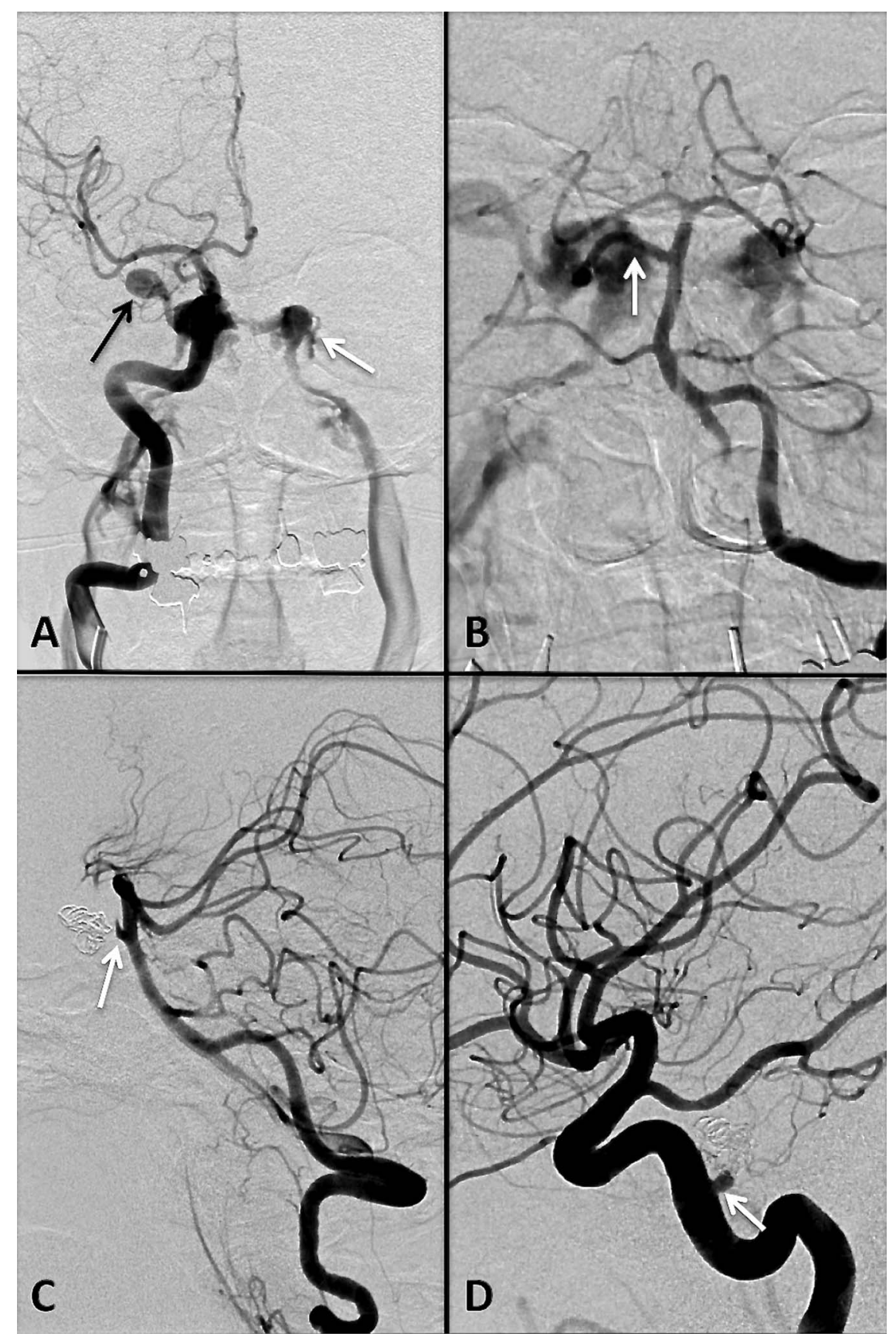

episode of valsalva. Physical examination demonstrated a rightsided objective orbital bruit, chemosis, increased intraocular pressure, and a sixth cranial nerve palsy. MR angiography (MRA) suggested an arteriovenous fistula involving the right cavernous sinus and an ipsilateral PTA, with subsequent catheter angiography demonstrating a right Saltzman type 2 trigemino-cavernous fistula (figure 1A, B), without evidence of an associated aneurysm or cortical venous reflux. Venous drainage was into an enlarged right superior ophthalmic vein (black arrow in figure 1A), contralateral cavernous sinus (white arrow in figure 1B), and bilateral inferior petrosal sinuses. A 6 F MPD Envoy catheter (Codman, Raynham, Massachusetts, USA) was positioned in the right internal carotid artery, through which an Excelsior SL-10 microcatheter (Stryker, Kalamazoo, Michigan, USA) was navigated over a Synchro-14 microwire (Stryker) across the fistula into the right cavernous sinus. Multiple platinum detachable coils were deployed in the right cavernous sinus, with post-embolization angiography demonstrating occlusion of both fistula and PTA (white arrows in figure $1 \mathrm{C}, \mathrm{D})$.

The patient remained asymptomatic for 10 months following treatment, at which time she developed recurrent right orbital symptoms. Time of flight MRA suggested fistula recurrence via a recanalized PTA (figure 2A) with catheter angiography demonstrating a small amount of stagnant arteriovenous shunting (figure 2B). Embolization of the recurrent fistula was attempted but could not be safely performed due to coil prolapse into the internal carotid artery. The patient's symptoms spontaneously resolved 2 months later and follow-up gadolinium enhanced MRA was suggestive of fistula closure (figure 2C). Slow stagnant flow through the recurrent fistula likely contributed to its subsequent spontaneous closure. The patient has been asymptomatic for over a year. 


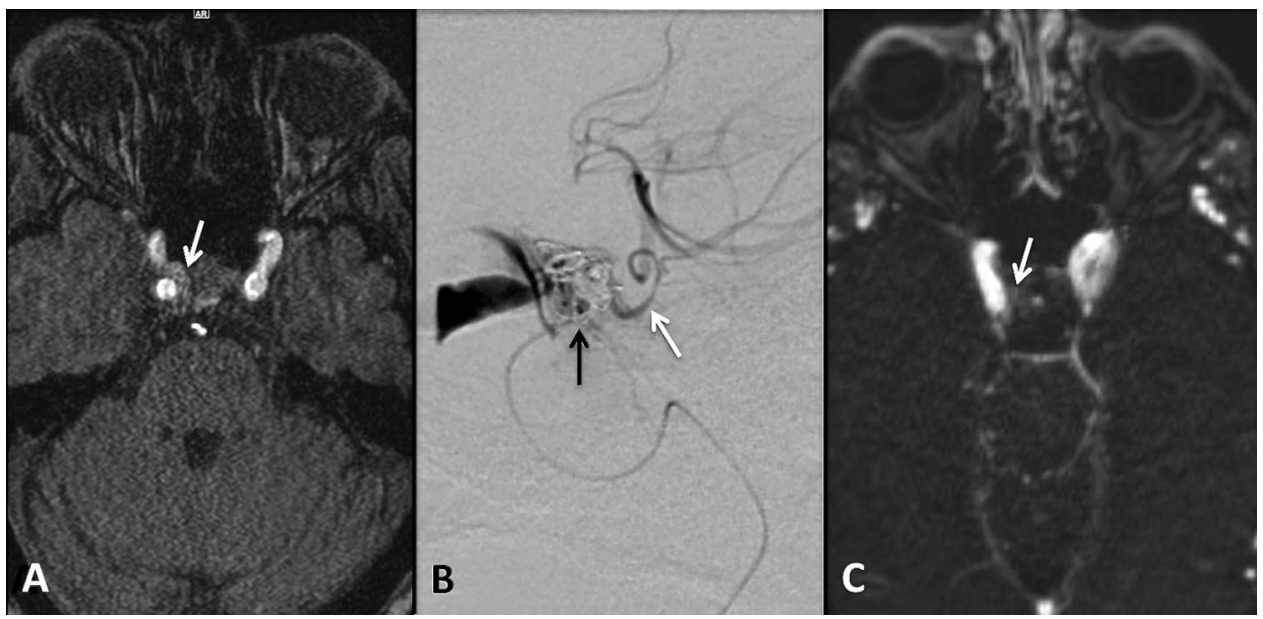

Figure 2 Three dimensional time of flight MR angiography $(A)$ performed several months following treatment is suggestive of recurrent arteriovenous shunting into the right cavernous sinus (white arrow $(A)$ ), which was confirmed on subsequent catheter angiography (B), including microcatheter injection (microcatheter tip, black arrow (B)) in the recanalized right persistent trigeminal artery (white arrow (B)). Additional embolization could not be performed at this time due to coil prolapse into the right internal carotid artery. A follow-up gadolinium enhanced MR angiogram (C) performed after spontaneous resolution of patient symptoms 2 months later demonstrates no evidence of residual arteriovenous shunting.

Figure 3 Anteroposterior (AP) and lateral views of a right internal carotid artery angiogram $(A, B)$ and $A P$ view of a left vertebral artery angiogram $(C)$ demonstrate a right trigemino-cavernous fistula with cross filing of the contralateral cavernous sinus and venous drainage into the bilateral inferior petrosal sinuses and internal jugular veins. The right persistent trigeminal artery is difficult to delineate on internal carotid artery injections due to rapid arteriovenous shunting (white arrow (B)), but is better seen on left vertebral artery injections (black arrows (C)). The site of fistulous communication between the persistent trigeminal artery (PTA) and cavernous sinus was confirmed by microcatheter injection in the aberrant vessel (D). The tip of the microcatheter (white arrow (D)), PTA (black arrow (D)), and tip of the basilar artery (arrowhead (D)) are clearly visualized.

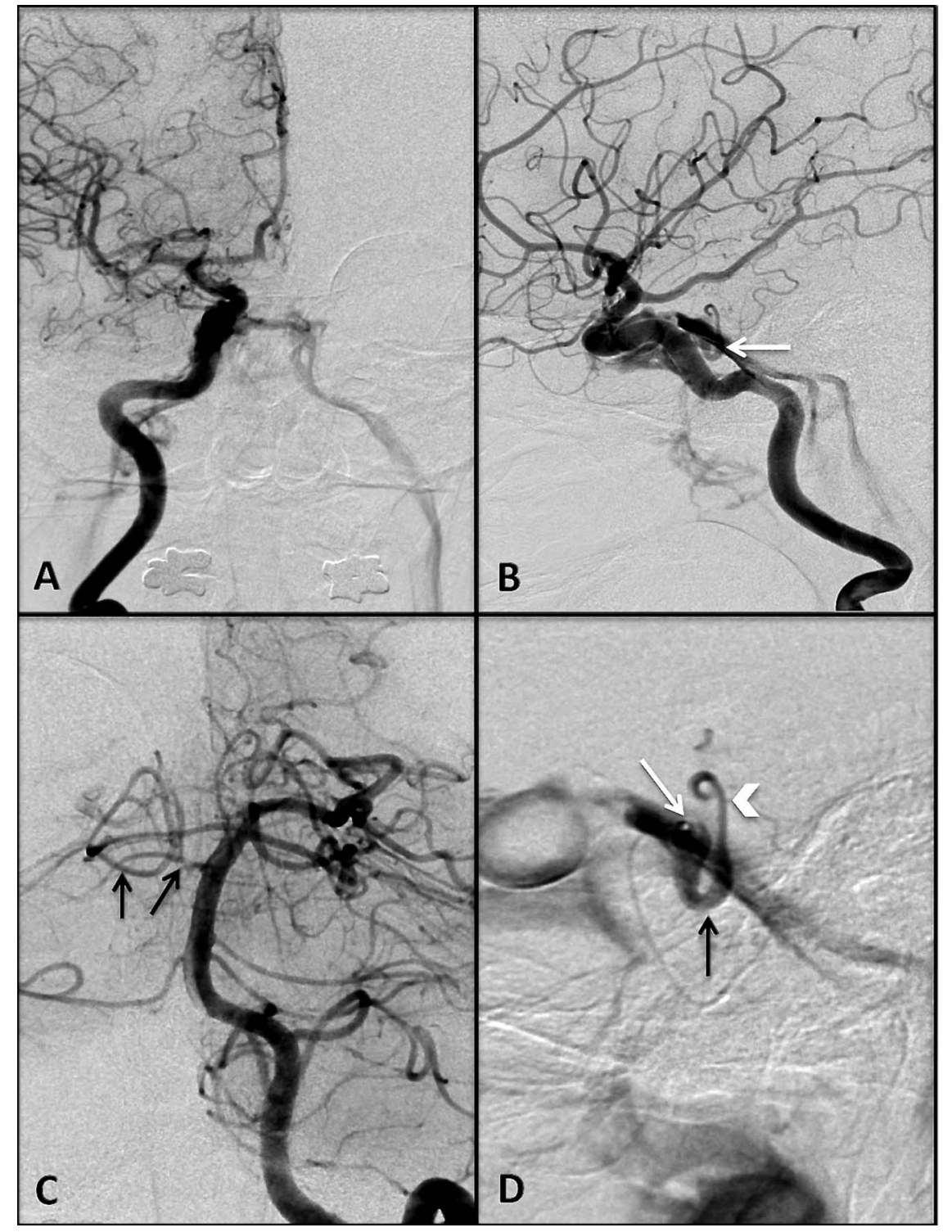


Figure 4 Lateral view of a left internal carotid artery angiogram (A) demonstrates a left trigemino-cavernous sinus fistula with extensive arteriovenous shunting with reflux into the ipsilateral orbital veins. Anteroposterior view of a left vertebral artery angiogram (B) more clearly demonstrates the left persistent trigeminal artery (PTA; white arrow (B)), including the left anterior inferior cerebellar artery (black arrow (B)) arising from the aberrant vessel. Coronal view from flat panel volume CT (C) better delineates the location of the fistula between the PTA (white arrow (C)) and cavernous sinus (black arrow (C)). Lateral view from a post embolization left internal carotid artery angiogram (D) performed approximately 3 months following treatment demonstrates successful fistula closure with coil mass in the left cavernous sinus (white arrow (D)) and preservation of the PTA (black arrow (D)).

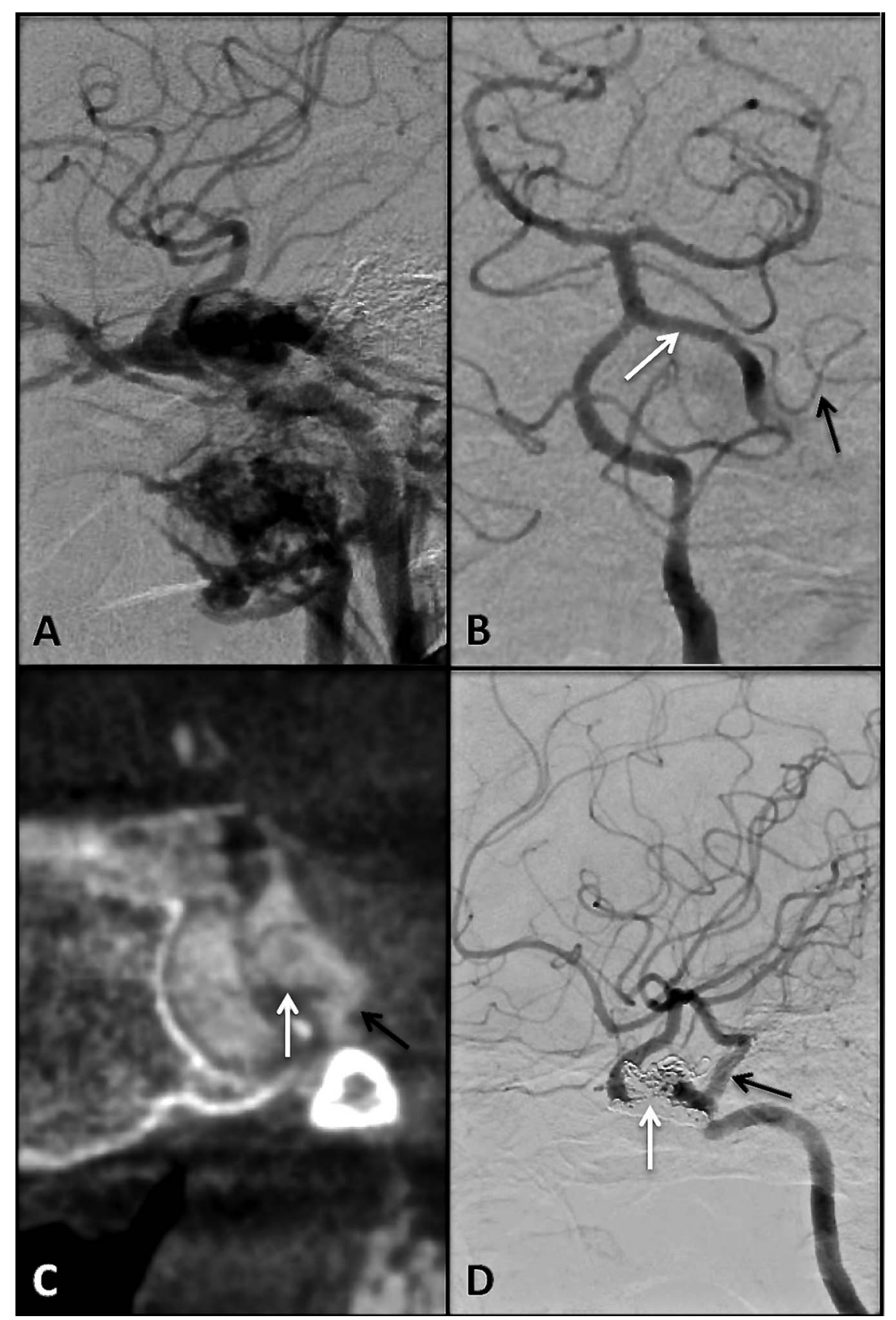

\section{Case No 2}

This woman insidiously developed right temple and eye pain as well as right pulsatile tinnitus following an episode of valsalva. Physical examination was otherwise unremarkable apart from a congenital right fourth cranial nerve palsy. Cerebral angiography demonstrated a Saltzman type 2 direct fistula between a right PTA and the ipsilateral cavernous sinus (figure 3A-D), without evidence of an associated aneurysm. Venous drainage was into contralateral cavernous and bilateral inferior petrosal sinuses, with no right orbital or cortical venous reflux.

The risks and benefits of endovascular treatment were explained in detail to the patient. However, she initially declined endovascular therapy, electing to clinically follow the lesion. One year after symptom onset, the patient's right temple and eye pain had resolved, although she continued to complain of pulsatile tinnitus, which varied in intensity. Further conservative management was felt to be reasonable at this time due to the persistent lack of cortical venous reflux on follow-up angiography and overall improvement in symptomatology. However, the patient elected to undergo palliative embolization of her fistula at an outside institution 6 months later for worsening pulsatile tinnitus, with subsequent fistula closure by transarterial PTA coil embolization.

\section{Case No 3}

This woman acutely developed left-sided orbital symptoms and pulsatile tinnitus following an infection of the head and neck. Physical examination demonstrated a left objective orbital bruit, ptosis, increased intraocular pressure, and an associated left third cranial nerve palsy. CTA suggested an arteriovenous fistula involving the left cavernous sinus and an ipsilateral PTA. The PTA was noted to give rise to a left anterior inferior cerebellar artery, consistent with a Saltzman variant. Cerebral angiography demonstrated the presence of a left trigemino-cavernous fistula (figure 4A, B) without evidence of an associated aneurysm. There was minimal cortical venous reflux. Due to rapid 
arteriovenous shunting, flat panel volume CT (figure 4C) and rotational three-dimensional DSA were utilized to precisely locate the fistulous communication to the proximal aspect of the PTA.

A 6 F MPD Envoy catheter was placed in the left vertebral artery. Through the guide catheter, an Excelsior SL-10 microcatheter was navigated over a Synchro-14 microwire and across the fistula into the left cavernous sinus. Multiple bare platinum detachable coils (Micrus, San Jose, California, USA) were then deployed in the cavernous sinus with post-embolization angiography demonstrating only trace residual arteriovenous shunting and preservation of the PTA. A 3 month follow-up angiogram demonstrated complete occlusion of the fistula (figure 4D). The patient has been asymptomatic now for over a year.

\section{DISCUSSION}

A PubMed database search using the keywords 'trigeminal artery fistula', performed July 1, 2013, resulted in 27 articles describing 30 cases (see online supplementary table S2).

Including our three patients, there have been 33 reported cases of trigemino-cavernous fistulas in the English literature. Only seven reported cases of trigemino-cavernous fistulas have had a documented associated PTA aneurysm (21\%) but six of these were in patients who had a spontaneous etiology of their fistula (30\% in this group). It can be speculated that high flow arteriovenous shunting may have obscured underlying aneurysms in other cases. ${ }^{1} 2$ Despite rapid arteriovenous shunting often resulting in flow reversal in the PTA, an associated posterior circulation vascular steal phenomenon has not been described in the literature. ${ }^{145}$

Twenty of the reported trigemino-cavernous fistulas were spontaneous (61\%) while the remaining 13 were traumatic (39\%). Spontaneous fistulas demonstrated a strong female predominance $(85 \%)$, and the majority of women were between 40 and 70 years of age. These have been associated with episodes of valsalva, as was the case for two of our patients, which may be due to transient elevations of intracranial or intra-arterial pressure. In contradistinction, traumatic trigemino-cavernous fistulas were more common in younger men (77\%), and all but three were younger than 40 years of age.

Thirty-two of 33 reported patients with trigemino-cavernous fistulas underwent treatment, while one declined therapy. A variety of endovascular and surgical techniques were used to achieve fistula closure, including surgical ligation and endovascular balloon embolization in earlier cases (three and 10 patients, respectively). More modern endovascular techniques have included transarterial or transvenous coil embolization of the cavernous sinus, PTA, and PTA aneurysm $(n=19)$. Two of these patients had treatment supplemented by embolization of fistulous elements using ethylene vinyl alcohol copolymer (Onyx; eV3, Irvine, California, USA) and n-butyl cyanoacrylate (Codman, Raynham, Massachusetts, USA), respectively.

Of the 19 patients undergoing primary endovascular coil embolization, 14 had definitive closure of the fistula following the initial treatment (74\%) while three more had eventual fistula closure documented by catheter angiography, with an overall $89 \%$ rate of successful fistula closure. The two remaining patients had fistula thrombosis suspected clinically or on noninvasive imaging, but not confirmed by catheter angiography. When including patients initially treated by endovascular detachable balloon embolization, 25 of 28 patients had eventual documented fistula closure by endovascular means alone (89\%). A summary of features, treatments, and outcomes of trigeminocavernous fistulas can be found in box 2 .
Box 2 Summary of features, treatments, and outcomes

of trigemino-cavernous fistulas

Etiology

- $20(61 \%)$ spontaneous

- $13(39 \%)$ traumatic

- Associated aneurysm visualized

- $7(21 \%)$ in all cases

- $6(30 \%)$ in fistulas presenting spontaneously

- Treatment

- $3(9 \%)$ surgical ligation

- $10(30 \%)$ balloon embolization of cavernous sinus, persistent trigeminal artery (PTA), and/or internal carotid artery

- $13(40 \%)$ transarterial coil embolization of cavernous sinus and/or PTA

- One case supplemented with Onyx embolization

- $5(15 \%)$ transvenous coil embolization of cavernous sinus

- One case supplemented by n-butyl cyanoacrylate embolization

- $1(3 \%)$ transarterial and venous coil embolization of cavernous sinus and PTA

- $1(3 \%)$ declined treatment

- Outcomes in treated cases

- $30(94 \%)$ patients with documented complete fistula closure

- $27(84 \%)$ with immediate fistula closure

○ $3(10 \%)$ with delayed fistula closure

- $2(6 \%)$ with fistula thrombosis suspected by examination or non-invasive imaging

- Preservation of PTA following treatment

- $15(47 \%)$ with vessel preserved

- 17 (53\%) with vessel sacrificed

- Complications

- $2(6 \%)$ patients with transient or temporary neurological deficits

There were two reported neurological complications in patients treated endovascularly. One patient who underwent surgical ligation of the fistula following failed balloon embolization developed a transient sixth cranial nerve palsy. The other patient had mild dysarthria and hemiparesis following transvenous and transarterial coil embolization of the involved cavernous sinus and PTA, which subsequently improved. Overall, the rate of neurological deficit following endovascular treatment of a trigemino-cavernous fistula was $7 \%$, and the mortality rate was $0 \%$.

The majority of reported cases of trigemino-cavernous fistulas have involved a Saltzman type 2 PTA, which some have argued should be preserved given its potential supply to the brainstem and trigeminal nerve. ${ }^{6} 8$ However, the PTA was preserved in only 15 of 32 treated patients (47\%). The low rate of ischemic complications in reported cases suggests that sacrifice of a Saltzman type 2 PTA can be performed if cavernous sinus embolization alone proves inadequate or difficult. Conversely, stenting of a Saltzman type 1 or variant PTA should be considered as needed to maintain vessel patency given its critical supply to the posterior circulation.

Our experience suggests that cross sectional imaging such as CT or MRA can be very helpful in delineating aberrant vasculature in patients suspected of having a direct fistula to 
the cavernous sinus. In the setting of a high flow fistula, an associated PTA or its aneurysm can be difficult to visualize on catheter angiography due to rapid arteriovenous shunting. Failure to recognize or fully delineate PTA anatomy prior to therapy may result in poor patient outcome, including posterior circulation ischemia in the setting of a Saltzman type 1 or variant PTA. Finally, contrast enhanced flat panel volume CT and/or three-dimensional rotational angiography should strongly be considered during the catheter angiogram if there is any uncertainty as to the precise location of the fistulous communication.

Although treatment is indicated for direct carotid-cavernous fistulas, our first two patients suggest that some trigeminocavernous fistulas may be conservatively followed. Specifically, those fistulas with mild symptoms and no cortical venous reflux may be candidates for such an approach. Periodic imaging follow-up would be required to exclude the development of the latter. In addition, small residual or recurrent fistulas after treatment may also be conservatively followed to allow time for spontaneous closure, potentially saving the patient from the risks of an additional embolization.

In summary, trigemino-cavernous fistulas present unique challenges in their diagnosis and treatment. Modern endovascular techniques are safe and effective at treating such fistulas but delineating the exact anatomy of the PTA is essential to treatment planning. The latter may be enhanced by cross sectional imaging prior to catheter angiography as well as flat panel volume CT and three-dimensional rotational angiography during the catheter angiogram. If the PTA demonstrates a Saltzman type 2 configuration, as was the case for our first two patients, occlusion of the vessel should be considered as warranted for fistula treatment. Finally, de novo or recurrent trigemino-cavernous fistulas without cortical venous reflux or intolerable symptomatology may be considered for conservative management.

\section{CONCLUSION}

Trigemino-cavernous sinus fistulas are rare direct fistulas between a PTA and the ipsilateral cavernous sinus, which require a thorough knowledge of the underlying aberrant vascular anatomy to both diagnose and safely treat. In the absence of cortical venous reflux, a thorough discussion of the risks and benefits of treatment, as well as alternatives including conservative management, should be presented to the patient.

Contributors TRM drafted the manuscript, analyzed the literature review, and assisted in revisions of the paper. GJ assisted in data collection, and drafting and revision of the manuscript. SM assisted in data collection and revision of the manuscript. MF assisted in data collection and drafting of the manuscript. BP assisted in revision of the manuscript. RH assisted in data collection. DG assisted in analysis of the literature review and revision of the manuscript.

Competing interests None.

Provenance and peer review Not commissioned; externally peer reviewed.

\section{REFERENCES}

1 Hurst RW, Howard RS, Zager E. Carotid cavernous fistula associated with persistent trigeminal artery: endovascular treatment using coil embolization. Skull Base Surg 1998;8:225-8

2 Meckel S, Spittau B, McAuliffe W. The persistent trigeminal artery: development, imaging anatomy, variants, and associated vascular pathologies. Neuroradiology 2013;55:5-16.

3 O'Uchi E, O'Uchi T. Persistent primitive trigeminal arteries (PTA) and its variant (PTAV): analysis of 103 cases detected in 16,415 cases of MRA over 3 years. Neuroradiology 2010;52:1111-19.

4 Qian CX, Ares C, Codere F, et al. Rupture of an aneurysm of the persistent trigeminal artery presenting as a carotid-cavernous sinus fistula. Orbit 2009;28:275-80.

5 Cheng WC, Wang AD. Carotid-cavernous sinus fistula associated with a primitive trigeminal artery. Neurosurgery 1990;27:802-5.

6 Matosevic $B$, Kiechl $S$, Werner $P$, et al. A case of a traumatic trigeminal-cavernous fistula occluded by coil embolization. J Neuroimaging 2011;21:280-2.

7 Kobayashi N, Miyachi S, Oi S, et al. Traumatic carotid-cavernous fistula associated with persistent primitive trigeminal artery treated by transarterial coil embolizationcase report. Neurol Med Chirurg 2011;51:37-40.

8 Yoshida M, Ezura M, Mino M. Carotid-cavernous fistula caused by rupture of persistent primitive trigeminal artery trunk aneurysm-case report. Neurol Med Chirurg 2011;51:507-11. 